OPEN ACCESS

Edited by:

Wei Cong,

Shandong University, Weihai, China

Reviewed by:

Yang Zou,

Lanzhou Veterinary Research Institute

(CAAS), China

Hongchao Sun,

Zhejiang Academy of Agricultural

Sciences, China

*Correspondence:

Jing Jiang

jiangjïnxiaoyao@163.com

He-Ting Sun

xiaofengsht@163.com

${ }^{\dagger}$ These authors have contributed equally to this work

Specialty section:

This article was submitted to

Clinical Microbiology,

a section of the journal

Frontiers in Cellular and

Infection Microbiology

Received: 04 September 2021 Accepted: 27 September 2021 Published: 18 October 2021

Citation:

Geng H-L, Ni H-B, Li J-H, Jiang J, Wang $W$, Wei $X-Y$, Zhang $Y$ and Sun $\mathrm{H}$-T (2021) Prevalence of Cryptosporidium spp. in Yaks (Bos grunniens) in China: A Systematic Review and Meta-Analysis. Front. Cell. Infect. Microbiol. 11:770612. doi: 10.3389/fcimb.2021.770612

\section{Prevalence of Cryptosporidium spp. in Yaks (Bos grunniens) in China: A Systematic Review and Meta-Analysis}

\author{
Hong-Li Geng ${ }^{1 \dagger}$, Hong-Bo $\mathrm{Ni}^{1+}$, Jing-Hao $\mathrm{Li}^{2}$, Jing Jiang ${ }^{3 *}$, Wei Wang ${ }^{4}, \mathrm{Xin} \mathrm{Yu} \mathrm{Wei}^{4}$, \\ Yuan Zhang ${ }^{5}$ and He-Ting Sun ${ }^{2 *}$
}

\begin{abstract}
${ }^{1}$ College of Veterinary Medicine, Qingdao Agricultural University, Qingdao, China, ${ }^{2}$ General Monitoring Station for WildlifeBorne Infectious Diseases, State forestry and Grass Administration, Shenyang, China, ${ }^{3}$ College of Life Sciences, Changchun Sci-Tech University, Shuangyang, China, ${ }^{4}$ College of Animal Science and Veterinary Medicine, Heilongjiang Bayi Agricultural
\end{abstract} University, Daqing, China, ${ }^{5}$ College of Animal Science and Technology, Jilin Agricultural University, Changchun, China

Cryptosporidium spp., the causative agent of cryptosporidiosis, can infect a variety of hosts. So far, there has been limited information regarding Cryptosporidium spp. infection in yaks (Bos grunniens). Here, we performed the first systematic review and meta-analysis for Cryptosporidium spp. infection in yaks in China. To perform the meta-analysis, five databases (Chinese National Knowledge Infrastructure (CNKI), VIP Chinese journal database, WanFang Data, PubMed, and ScienceDirect) were employed to search for studies related to the prevalence of Cryptosporidium spp. in yaks in China. The total number of samples was 8,212, and the pooled Cryptosporidium spp. prevalence in yaks was estimated to be $10.52 \%$ (1192/8012). The prevalence of Cryptosporidium spp. in yaks was 13.54\% (1029/5277) and 4.49\% (148/2132) in northwestern and southwestern China, respectively. In the sampling year subgroups, the prevalence before 2012 (19.79\%; 650/2662) was significantly higher than that after 2012 (6.07\%; 437/4476). The prevalence of Cryptosporidium spp. in cold seasons (20.55\%; 188/794) was higher than that in warm seasons (4.83\%; 41/1228). In the age subgroup, the yaks with age $<12$ months had a higher prevalence (19.47\%; 231/1761) than that in yaks with age $\geq 12$ months (16.63\%; 365/2268). Among 12 Cryptosporidium spp. species/genotypes, the C. bovis had the highest prevalence. Moreover, the effects of geography (latitude, longitude, precipitation, temperature, and altitude) and climate on Cryptosporidium spp. infection in yaks were evaluated. Through analyzing the risk factors correlated with the prevalence of Cryptosporidium spp., we recommend that effective management measures should be formulated according to the differences of different geographical factors, in order to prevent cryptosporidiosis and reduce economic losses in yaks in China.

Keywords: Cryptosporidium spp., yaks, China, meta-analysis, prevalence, zoonosis 


\section{INTRODUCTION}

Cryptosporidium spp. is an opportunistic protozoan that parasitizes the mucosal epithelial cells of gastrointestinal tract in animals (Wang et al., 2019a). Cryptosporidium spp. has a wide range of hosts, including cattle, cats, birds and human (Bhat et al., 2019). The transmission routes for Cryptosporidium spp. include a direct contact with infected animals, contaminated water or food, and fecal-oral route (Qin et al., 2014; Ryan et al., 2016; Yildirim et al., 2020). In general, the infection of Cryptosporidium spp. in individual was asymptomatic. However, severe symptoms may be induced in immunocompromised individual (Desai, 2020).

The average altitude of yaks' (Bos grunniens) habitats is around 3,000 meters above sea level (Lan et al., 2020). The main habitats for yaks are in Tibet municipality, Qinghai Province, Gansu Province, and Sichuan Province (Wang et al., 2019b). Qinghai Province, which was identified to be the largest population of yaks in the world, has approximately 5 million yaks (Wang et al., 2018). So far, 38 species and over 70 genotypes of Cryptosporidium spp. have been identified (Deng et al., 2020). Twelve Cryptosporidium spp. species/ genotypes have been identified in yaks, including C. bovis, C. ryanae, C. baileyi, C. andersoni, C. parvum, C. hominis, C. canis, C. struthionis, C. xiaoi, and C. ubiquitum (Ma et al., 2014b; Qi et al., 2015; Wang et al., 2018). More importantly, some of them, such as C. parvum, C. hominis, and C. ubiquitum, were also frequently found in humans (Widmer, 2009; Li et al., 2014; Ryan et al., 2016), and the infection rate is $36.4 \%, 9.3 \%$ and $1.6 \%$ (Guy et al., 2021). Cryptosporidium may cause fatal persistent diarrhea in infants and people with weakened or immune function and cognitive development, thus representing a public health threat (Xiao et al., 2004). The droppings of yaks that infected with Cryptosporidium spp. can be washed away by rain, thus resulting in an influx of Cryptosporidium spp. oocysts into the local source of water. The herdsmen and yaks, who live on the plateau, have a high probability to share the source of water. Thus, the yaks infected with Cryptosporidium spp. could bring the pathogen to herdsmen through the shared water (Wang et al., 2018). Cryptosporidium spp. infection in yaks can cause a loss of appetite, diarrhea, and other symptoms, which leads to a reduced resistance to the disease (Huang et al., 2014; Li et al., 2016a; Gong et al., 2017). The people living on the plateau can obtain various daily necessities (e.g., milk and beef) from yaks. Thus, the yaks are one of the important economic resources for the local people, leading to a direct correlation of yak's health and economy (Mi et al., 2013). So far, there has been no effective drugs or available vaccines for preventing and controlling cryptosporidiosis (Gao, 2012; Ikiroma and Polloc, 2021). The prevention of cryptosporidiosis is an important approach for reducing losses to the breeding industry.

Currently, a systematic evaluation and analysis for cryptosporidiosis in yaks is absent. Thus, it is essential to carry out a systematic evaluation and meta-analysis based on the existing literatures. In this study, our study aim was to analyze the epidemic status of cryptosporidiosis among yaks in China,

Abbreviations: VIP, VIP Chinese Journal Databases; CNKI, China National Knowledge Infrastructure; WanFang, WanFang Databases. evaluate and discuss the corresponding risk factors that contribute to Cryptosporidium spp. infection in yaks.

\section{METHODS}

\section{Systematic Search Strategy}

This paper was prepared according to the PRISMA guidelines for the design and analysis of selected qualified studies (Table S1). A literature search was conducted to identify articles published from the inception to January 18, 2021. The aim was to obtain all articles in Chinese and English with topics of Cryptosporidium spp. infection in yaks in China. The articles were collected from five databases, including China National Knowledge Infrastructure (CNKI), VIP Chinese Journals Database, Wanfang Data, PubMed, and ScienceDirect. The keywords "yak" and "Cryptosporidium" were used for searching on the databases CNKI, VIP Chinese Journals Database, Wanfang Data, and ScienceDirect. The MeSH terms "Cryptosporidium", "yak" and "China", and their entry terms, such as "Bos indicus", "Zebu”, "Bos taurus", "Domestic Cow", "Domestic Cows", "Bos grunniens", and "Cryptosporidium" were used for searching on PubMed. The boolean operators "AND" and "OR" were used to connect $\mathrm{MeSH}$ terms and the entry terms, respectively. Finally, the search formula "((Cryptosporidium) OR Cryptosporidiums) AND ((()((()(((yak) OR Bos indicus) OR Zebu) OR Zebus) OR Bos taurus) OR Cow, Domestic) OR Cows, Domestic) OR Domestic Cow) OR Domestic Cows) OR Bos grunniens) OR Yak) OR Yaks $))))$ AND ((((((China) OR People's Republic of China) OR Mainland China) OR Manchuria) OR Sinkiang) OR Inner Mongolia)" was used for searching on PubMed. The Endnote (X9.2 version) was employed to collate information of obtained articles.

\section{Data Extraction and Exclusions}

The inclusion criteria for our systematic review and metaanalysis were as follows: (1) the subjects of the study were limited to yaks; (2) the detection of Cryptosporidium spp. was at least carried out by nucleic acid or pathogen detection methods, such as PCR, ELISA or microscopy; (3) the selected articles should contain the information of sample number, positive number, and detection site; (4) the article should contain a full-text with complete data; (5) studies must be designed for a cross-sectional extension; (6) the sample should come from a separate yak (not a mixed sample).

The extracted data included the first author, the year of publication, the province where the study performed, sample collection time, age and gender of yak, detection method, sampling seasons, geographical location (latitude and longitude), relative humidity, annual average temperature, annual precipitation, method type, total number of samples, number of positive samples, and data score. According to the report by Fan and colleagues, the climate of China's plateau is unique, with the warm weather from June to October and the cold weather from November to May (Fan et al., 2011). Therefore, this division method was used to classify seasonal subgroups in this study. Our database was constructed by using Microsoft Excel (version 16.32). 
Two reviewers independently extracted and recorded data from each selected research. The differences derived from reviewers or uncertainty about the qualifications of the research were further assessed by another author of this paper.

\section{Quality Assessment}

The standardized data collection table was used for data extraction according to the research purpose and inclusion criteria. The article quality was evaluated based on the Grading of Recommendations Assessment reported previously (Guyatt et al., 2008). The scoring criteria of data scoring items were as follows: (1) there was a detailed sampling time-point; (2) there was a specific sampling location; (3) the number of samples was over than 200; and (4) there were more than three risk factors. According to the above scoring criteria, 1 point was given for each item, and the total score of each item was added up to get the total score of the article. The total score was identified to be high quality for 3-4 points, medium quality for 2 points, and low quality for $0-1$ points.

\section{Statistical Analyses}

The meta package in R software version 4.0.3 ("R core team, R: A language and environment for statistical computing" R core team 2018) was used to analyze the data in this study (Li et al., 2020a). The $\mathrm{W}$-value close to 1 and the $P$-value greater than 0.05 is identified to be close to the Gaussian distribution criterion. The double-arcsine transformation (PFT) method was chosen for data conversion (Table 1). The heterogeneity among studies was predicted by Cochran's Q-value (represented by $X^{2}$ and $P$-value) and $I^{2}$ statistics. Cochran's Q $\left(X^{2}\right.$ and $P$-value $)$ and $I^{2}$ statistics were employed to predict the inter-study heterogeneity. The random effect model was chosen for an analysis, according to the heterogeneity of the included articles (Ni et al., 2020). Forest plots were used for a comprehensive analysis. Funnel plot and Egger's test were used to evaluate the publication bias. The stability of the study was evaluated by the trim and filling test, and sensitivity analysis (Wang et al., 2020).

The potential sources of heterogeneity were further studied by subgroup analysis and meta regression analysis. The individual and multivariate model factors were analyzed to determine the factors contributing to the heterogeneity. The survey factors included the sampling year (before 2012 vs. after 2012), region (Northwestern China vs. Southwestern China), province (Qinghai province vs. other provinces), diagnostic method

TABLE 1 | Normal distribution test for the normal rate and the different conversion of the normal rate.

\begin{tabular}{lcc}
\hline Conversion form & w & $\boldsymbol{P}$ \\
\hline PRAW & 0.86549 & 0.009798 \\
PLN & $\mathrm{NaN}$ & $\mathrm{NA}^{*}$ \\
PLOGIT & $\mathrm{NaN}$ & $\mathrm{NA}^{*}$ \\
PAS & 0.93191 & 0.1681 \\
PFT & 0.93356 & 0.1808 \\
\hline
\end{tabular}

"PRAW": original rate; "PLN": logarithmic conversion; "PLOGIT": logit transformation;

"PAS": arcsine transformation; "PFT": double-arcsine transformation;

"NaN": meaningless number; "NA": missing data.
(Immunofluorescence technique (IFA) vs. other methods), age (age $<12$ months $v s$. age $\geq 12$ months), season (cold seasons vs. warm seasons), genotype (Cryptosporidium bovis vs. other genotypes), the quality level of the included publications (high quality $v s$. others), longitude $\left(95-100^{\circ} v s\right.$. others), latitude ( $>35^{\circ} v s$. others), altitude $\left(<95^{\circ} v s\right.$. others), average annual precipitation $(<300 \mathrm{~mm}$ vs. $>300 \mathrm{~mm})$, average annual temperature $\left(<1^{\circ} \mathrm{C} v s\right.$. others), average annual humidity $(<55 \%$ $v s . \geq 55 \%)$, altitude $(<3000 \mathrm{~m} v s .>3000 \mathrm{~m})$, and climate (plateau mountain vs. others).

\section{RESULTS}

\section{Search Results}

Through searching on five databases, 1,006 relevant articles were screened out for further analyses. According to the selection criteria described in section " 2.2 ", the uncertain articles were excluded by checking the abstracts and/or full-text. Finally, 49 out of 1,006 articles were selected. Among of the selected articles, four were repeated publications, ten were not research objects, one was overview article and letter, and fourteen were removed due to an incomplete or unclear information. Thus, a total of 20 articles were included in this study (Figure 1).

\section{Qualification Studies and Publication Bias}

Consequently, the included articles covered four provinces. Among the 20 studies, the total number of samples and positive number was 8,212 and 1,192 , respectively (Table 2). Based on the quality standard, fourteen articles were of high quality ( 3 or 4 points), five were of medium quality ( 2 points), and one was of low quality (1 point; Table 2 and Table S1).

In the selected studies, the forest plot measurement demonstrated the degree of heterogeneity (Figure 2). According to the funnel chart, we found that the distribution of dots was not completely symmetrical, which might be explained by publication bias or small sample bias (Figures 3, 4). No supplementary study was found by the trim and filling test. The Egger test was used to assess the potential publication bias in the analysis, and the $P$ value greater than 0.05 indicated that no publication bias was present in the data (Figure 5). Sensitivity test indicated that the recombined data were not significantly affected by any study that was excluded (Figure 6). These results verified rationality and reliability of our analyses.

\section{Results of the Meta-Analysis}

From 2001 to 2021, the total prevalence of Cryptosporidium spp. in yaks in China was $10.52 \%$ (95\% CI: 5.64-16.63; Table 2). In region group, the higher prevalence was detected in northwestern China (13.54\%, 95\% CI: 7.10-21.58); than southwestern China (Table 2). In the covered four provinces of the meta-analysis, Qinghai province had the highest prevalence of $14.17 \%$ (95\% CI: 7.34-22.70), and Gansu province and Tibet municipality had the lowest prevalence of 5.98\% (95\% CI: 2.29-11.12) and 6.03\% (95\% CI: 4.56-21.34), respectively (Table 3 ). To further identify sources of 


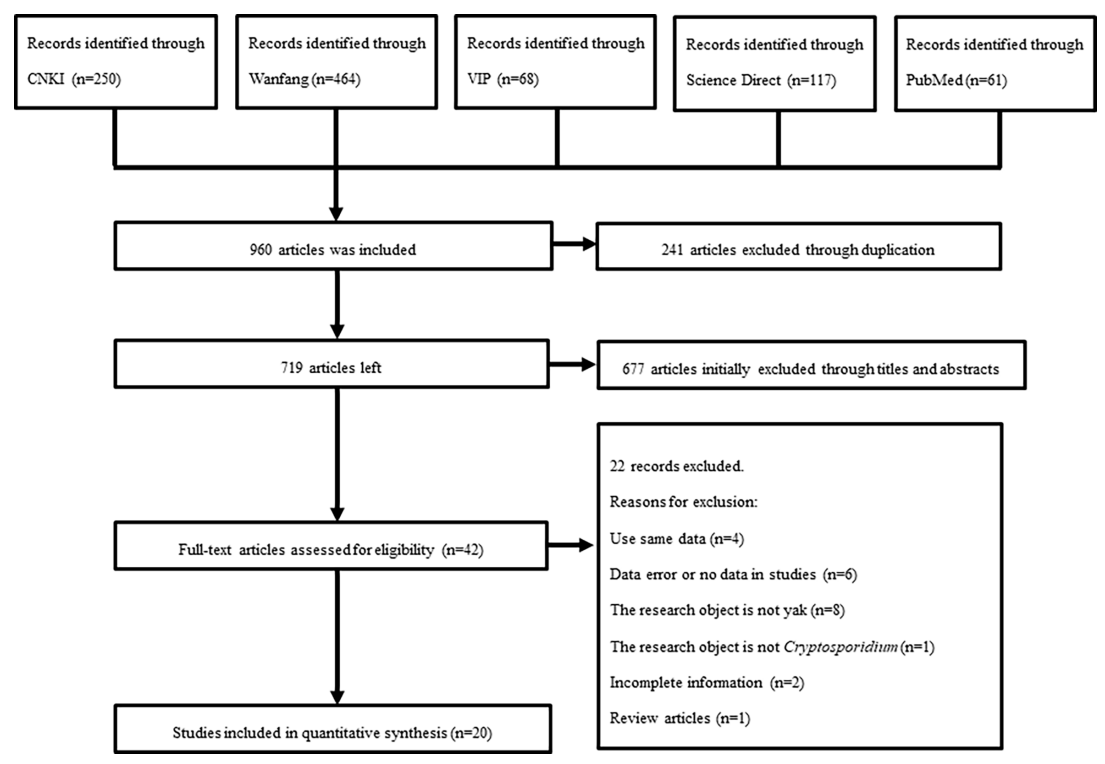

FIGURE 1 | Flow diagram of literature search and selection.

heterogeneity, we analyzed subgroups of season, age, sampling year, detection methods, detailed geographic, and climatic factors. Sampling year was a risk factor for Cryptosporidium spp. infection in yaks $(P<0.05$; Table 2$)$. The prevalence of Cryptosporidium spp. in yaks before 2012 was $19.47 \%$ (95\% CI: 10.25-30.56), and 6.07\% (95\% CI: 1.64-12.91) in yaks after 2012 respectively (Table 2). Among 12 Cryptosporidium spp. species/ genotypes, the $C$. bovis has the highest prevalence $(0.34 \%, 173 /$ 4277, 95\% CI: $0.16-0.25)$, followed by C. andersoni ( $0.25 \%, 100 /$ 4277, 95\% CI: 0.19-0.32) and C. parvum (0.25\%, 95\% CI: 0.17-
0.35; Table 4). The prevalence of C. baileyi, C. ubiquitum, and $C$. xiaoi were the lowest $(0.02 \%, 1 / 4277,95 \%$ CI: $0.00-0.40 ; 2 / 4277$, 95\%CI: $0.00-0.10 ; 1 / 4277,95 \%$ CI: $0.00-0.10$; Table 4). The information for subgroups analysis of geographical latitude included latitude range $\left(>35^{\circ} ; 14.68 \%\right.$, 95\% CI: 6.19-25.85), longitude range $\left(<95^{\circ} ; 6.03 \%, 95 \%\right.$ CI: $\left.0.37-16.76\right)$, precipitation range (> $300 \mathrm{~mm} ; 12.27 \%$, 95\% CI: 6.66-19.24), temperature range $\left(<1^{\circ} \mathrm{C} ; 19.96 \%, 95 \% \mathrm{CI}: 10.49-31.38\right)$, humidity range ( $\geq 55 \% ; 12.36 \%, 95 \%$ CI: 4.74-22.80), and altitude range (<3000 m; 13.15\%, 95\% CI: 5.38-23.60; Table 5).

TABLE 2 | Pooled prevalence of Cryptosporidium infection in yaks in China.

\begin{tabular}{|c|c|c|c|c|c|c|c|c|c|c|}
\hline Variable & Category & No. studies & No. examined & No. positive & $\%\left(95 \% \mathrm{Cl}^{\star}\right)$ & \multicolumn{3}{|c|}{ Heterogeneity } & \multicolumn{2}{|c|}{ Univariate meta-regression } \\
\hline \multirow[t]{2}{*}{ Season } & Cold & 5 & 794 & 188 & $20.55 \%(9.76-33.96)$ & 63.24 & $<0.01$ & 95.3 & 0.012 & 0.331 (0.074 to 0.588$)$ \\
\hline & Warm & 5 & 1228 & 41 & $4.83 \%$ (0.12-14.52) & 0.14 & $<0.01$ & 0.0 & & \\
\hline \multirow[t]{2}{*}{ Age } & $<12$ months & 11 & 1761 & 231 & $19.47 \%(10.25-30.56)$ & 241.34 & $<0.01$ & 95.9 & 0.659 & $0.042(-0.145$ to 0.229$)$ \\
\hline & $\geq 12$ months & 6 & 2268 & 365 & $16.63 \%(7.84-27.84)$ & 191.62 & $<0.01$ & 97.4 & & \\
\hline Collection & Before 2012 & 7 & 2662 & 650 & 19.79\% (9.34-32.83) & 290.71 & $<0.01$ & 97.9 & 0.025 & 0.214 (0.027 to 0.400$)$ \\
\hline \multirow{3}{*}{ Method } & Microscopy & 7 & 1884 & 206 & $12.00 \%(4.04-23.29)$ & 248.58 & $<0.01$ & 97.6 & & \\
\hline & ELISA & 2 & 1229 & 369 & $12.44 \%(0.00-58.23)$ & 127.99 & $<0.01$ & 99.2 & & \\
\hline & IFA & 3 & 560 & 45 & $6.52 \%(0.16-18.65)$ & 24.98 & $<0.01$ & 92.0 & & \\
\hline \multirow[t]{2}{*}{ Region* } & Northwestern & 15 & 5277 & 1029 & 13.54\% (7.10-21.58) & 826.49 & $<0.01$ & 98.3 & 0.093 & $0.160(-0.026$ to 0.346$)$ \\
\hline & Southwestern & 6 & 2132 & 148 & $4.49 \%(0.60-11.22)$ & 139.93 & $<0.01$ & 96.4 & & \\
\hline Quality* level & High & 14 & 6951 & 1056 & $12.14 \%$ (6.00-20.02) & 1041.69 & $<0.01$ & 98.8 & 0.426 & $0.081(-0.118$ to 0.280$)$ \\
\hline
\end{tabular}

$\mathrm{Cl}^{*}$, Confidence interval; $N A^{*}$, not applicable; $P$-value ${ }^{*} P<0.05$ is statistically significant.

Region*: Northwestern China: Qinghai, Gansu; Southwestern China: Sichuan, Tibet.

Quality* : High: 4 or 3 points; Middle: 2 points; Low: 1 point. 


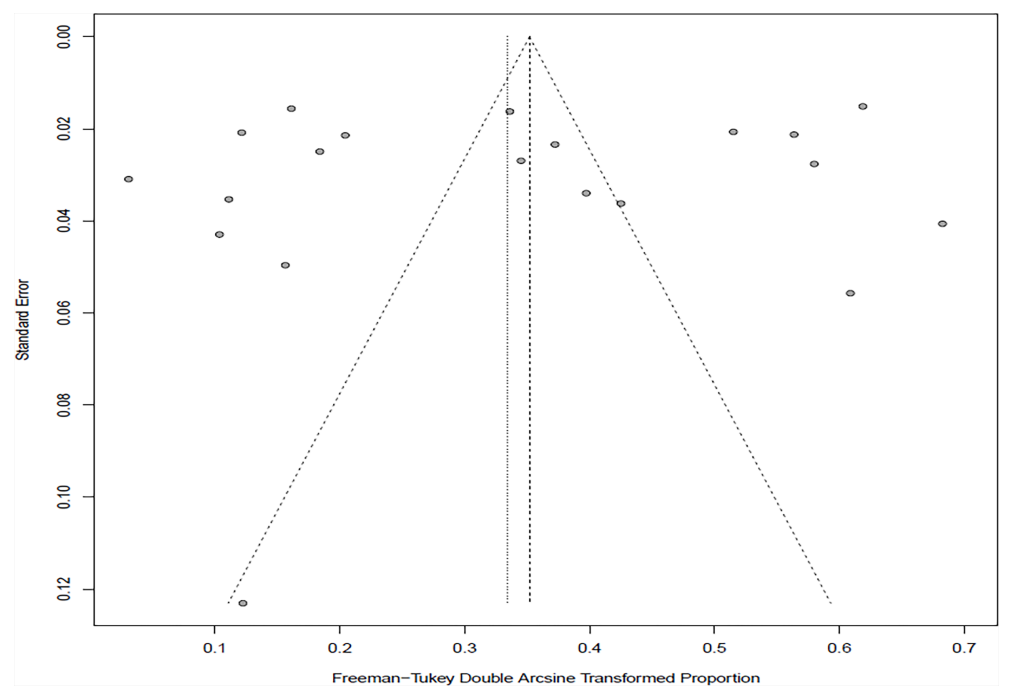

FIGURE 2 | Funnel plot with pseudo 95\% confidence interval for publication bias test.

\section{DISCUSSION}

Cryptosporidium spp. can cause economic losses in animal husbandry, and bring a great threat to human health (Ouakli et al., 2018; Pumipuntu and Pirate, 2018). Therefore, it is essential to understand the prevalence of Cryptosporidium spp. in its hosts. A systematic review and meta-analysis of Cryptosporidium spp. prevalence among yaks in China was performed in this study. In 2012 and 2013, China issued the mid to long term animal disease prevention plan (2012-2020) and the National Development Plan for Beef and Mutton Production (2013-2020) to strengthen the prevention and control for animal diseases (Gong et al., 2020; Wei et al., 2021). Therefore, the year "2012" is taken as the cut-off timepoint. After an introduction of the above policies, the effective prevention and control measurements might be one reason for the decreased prevalence of Cryptosporidium spp. after 2012 (General Office of the State Council, 2012).

In general, Cryptosporidium spp. prefers to live in a warm and humid environment, such as southwestern regions (Jagai et al., 2009; Taghipour et al., 2020). However, the prevalence of Cryptosporidium spp. in the northwestern regions was reported to be higher than that in the southwestern regions. We found that most of the articles retrieved in the southwestern regions

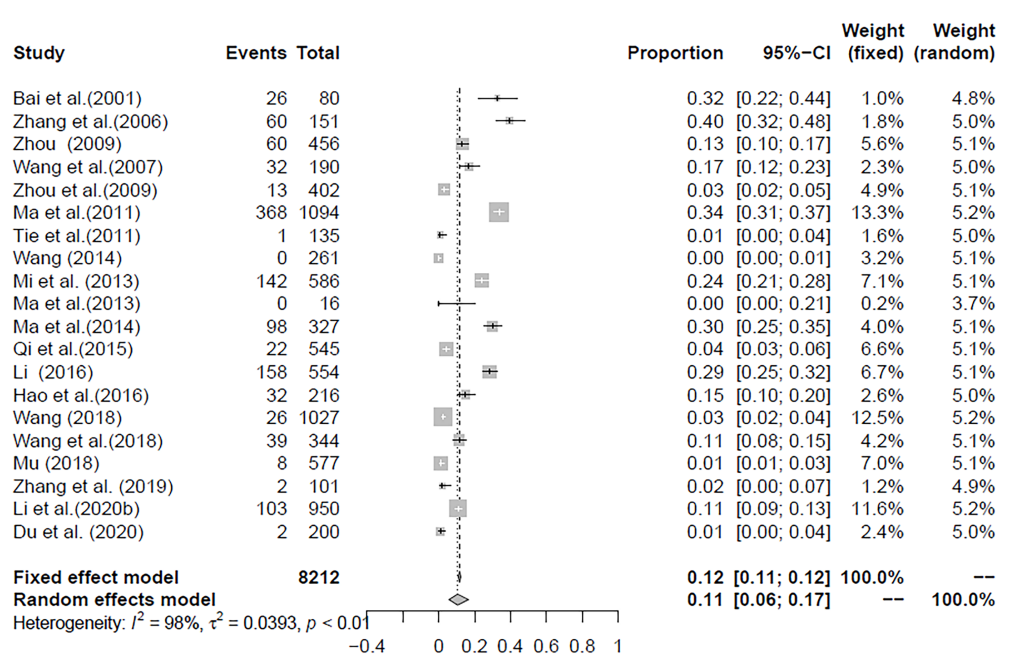

FIGURE 3 | Forest plot of Cryptosporidium prevalence in yaks in China. 


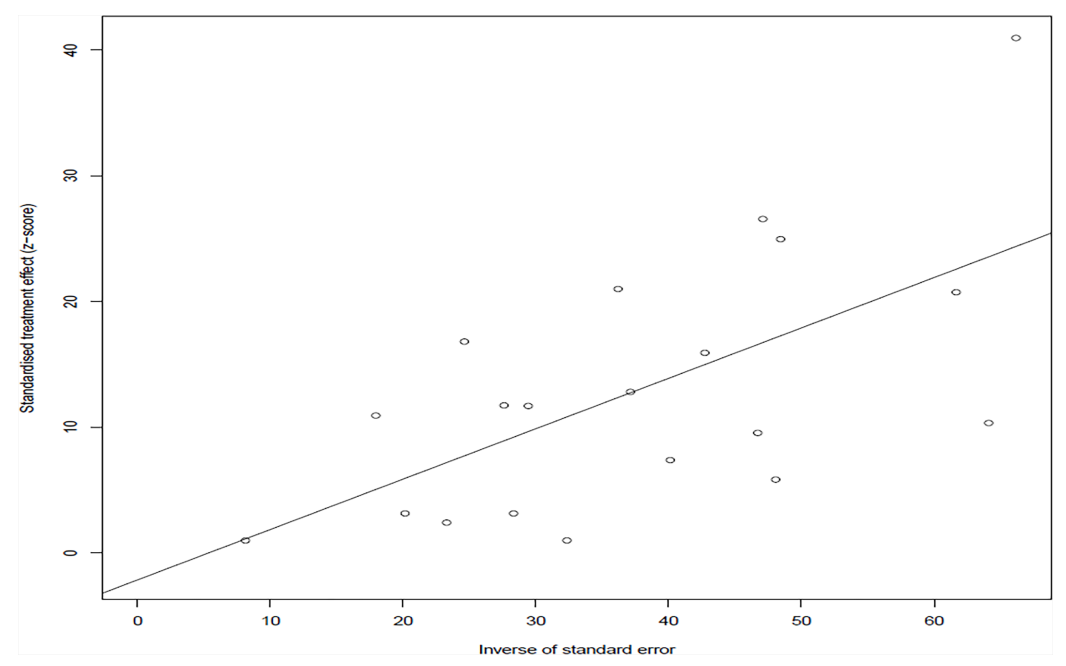

FIGURE 4 | Publication bias of included studies by Egger' test.

were from Qinghai province (Figure 7). Qinghai province had a significant effect on the results of northwestern China. Meanwhile, the infection rate of Cryptosporidium spp. in Qinghai province was found to be the highest among the analyzed provinces. Several studies showed that the prevalence of Cryptosporidium spp. in other animals was also at a high level in Qinghai province. For instance, the prevalence of Cryptosporidium spp. is identified to be $22.8 \%$ and $39.02 \%$ in sheep and goats, respectively (Karanis et al., 2007; Niu and Ma, 2007; Ma et al., 2010; Ma et al., 2013). Some of the water in Qinghai province contains high concentration of Cryptosporidium spp. oocysts (Ma et al., 2014a; Ma et al., 2019), and the infected animals were also potential factors inducing water pollution. The oocysts in the environment were difficult to be eliminated, thus resulting in an increased Cryptosporidium spp. infection rate in yaks through ingesting contaminated water (Li et al., 2016b; Li et al., 2019). This may lead to an increase of Cryptosporidium spp. infection in yaks. Multiple factors, such as climate change, animal husbandry practices, and parasite control measures, may cause various prevalence in different geographic regions (Taghipour et al., 2020). The latitude and longitude of Qinghai province are " $31^{\circ}$ $36^{\prime}-99^{\circ} 19^{\prime \prime \prime}$ and " $89^{\circ} 35^{\prime}-103^{\circ} 04^{\prime \prime}$, respectively. At the same time, we found that the areas with latitude $>35^{\circ}$ and longitude of 95$100^{\circ}$ were also located in Qinghai province, and the infection rate was high (Table 4). Qinghai province has a typical continental

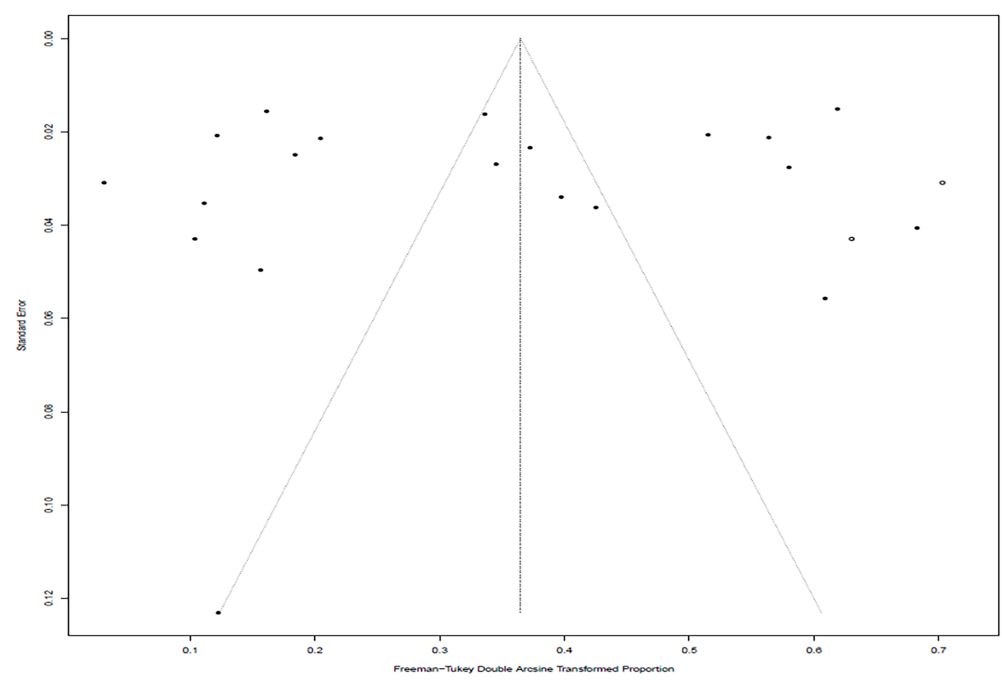

FIGURE 5 | The trim and filling test. 


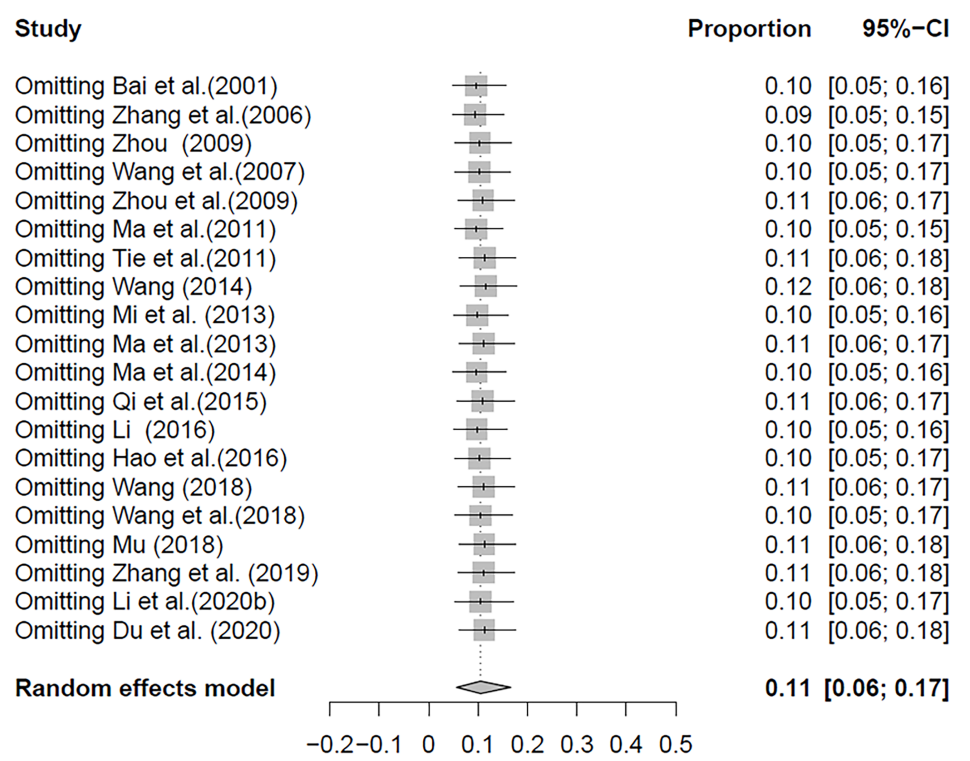

FIGURE 6 | Sensitivity test.

plateau climate (Zhang, 2010) that is high altitude, low temperature, and unpredictable climate (Wei et al., 2015; Zhang et al., 2019). The same characteristics were also observed in our climate subgroup analysis. The prevalence of Cryptosporidium spp. in the continental plateau climate was higher than that in other subgroups.

The prevalence of Cryptosporidium spp. in yaks $<12$ months was higher than that $\geq 12$ months based on our data. The age of sexual maturity of the yak is about 12 months, so the age "12 months" is taken as the cut-off age-point (Wen, 1988). The maternal antibodies obtained from colostrum in young yaks disappear approximately in 2-6 months, therefore, the immunity may decrease and then result in an increased morbidity (Sareyyüpoğlu et al., 2019; Wang et al., 2020). The prevalence of Cryptosporidium spp. was slightly lower in the younger yaks.

To date, a total of 12 Cryptosporidium spp. species/genotypes were identified in yaks. Among these species/genotypes, C. parvum, C. hominis, and C. ubiquitum were identified in humans, which has caused a widespread concern (Widmer, 2009; Li et al., 2014; Ryan et al., 2016). Interestingly, co-infection of two species/genotypes ( $C$. ryanae and C. bovis or C. parvum and C. bovis) was also found in yaks (Mi et al., 2013; Ma et al., 2014b), suggesting that the environment might be contaminated by more than one Cryptosporidium spp. species/genotype. The present study found that $C$. bovis had the highest prevalence in the investigated yaks.
C. bovis is one of the main genotypes that cause cryptosporidiosis in cattle (Wang et al., 2017) and C. bovis has been found to be the most prevalent species in pre-weaned calves (Wang et al., 2011; Murakoshi et al., 2012; Zhang et al., 2013). Other studies have also confirmed C. bovis was the dominant species in cattle (Mi et al., 2013; Ma et al., 2014b).

In the subgroup of precipitation, the prevalence of Cryptosporidium spp. at altitude $<3000 \mathrm{~m}$ was higher than that at altitude $>3000 \mathrm{~m}$. Additionally, the temperature was usually high at the low altitude. Previous studies showed that cryptosporidiosis mainly occurred in warm and humid seasons (Lou, 2016). In the subgroups of precipitation and humidity, we found that the prevalence of Cryptosporidium spp. in precipitation (> $300 \mathrm{~mm}$ ) and humidity (> 55\%) environment was also high. Thus, our data were in line with previous findings (Taghipour et al., 2020).

The prevalence of Cryptosporidium spp. in the cold weather was higher than that in the warm weather, owing to a generally lower temperature on the plateau (Taghipour et al., 2020). Due to the special physiological characteristics of the yak, most of the yaks are grazing in the resource-rich plateau grasslands (Fu et al., 2018). The forage has a low nutrient content in the cold weather, which does not meet the nutrients required by yaks. This causes a loss of body weight and a decreased immunity of yaks, and thus increasing the probability of Cryptosporidium

TABLE 3 | Pooled Cryptosporidium prevalence in yaks in various provinces.

\begin{tabular}{|c|c|c|c|c|c|c|}
\hline Provinces & Regions & No. Studies & No. tested & No. positive & Prevalence (\%) & $95 \% \mathrm{Cl}$ \\
\hline Qinghai & Northwestern & 14 & 5160 & 1022 & $14.17 \%$ & $7.34-22.70$ \\
\hline Sichuan & Southwestern & 3 & 561 & 33 & $3.15 \%$ & $0.00-17.38$ \\
\hline Tibet & Southwestern & 3 & 1571 & 115 & $6.03 \%$ & $4.56-21.34$ \\
\hline Gansu & Northwestern & 1 & 117 & 7 & $5.98 \%$ & $2.29-11.12$ \\
\hline
\end{tabular}


TABLE 4 | The species/genotype of Cryptosporidium in yaks was detected by PCR.

\begin{tabular}{|c|c|c|c|c|c|c|c|c|c|}
\hline Category & No. studies & No. examined & No. positive & $\%\left(95 \% \mathrm{Cl}^{\star}\right)$ & \multicolumn{3}{|c|}{ Heterogeneity } & \multicolumn{2}{|c|}{ Univariate meta-regression } \\
\hline C. ryanae & 10 & 4277 & 117 & $0.20 \%(0.16-0.25)$ & 120.12 & $<0.01$ & 92.5 & 0.104 & $0.0183(-0.0037$ to 0.0403 \\
\hline C. baileyi & 1 & 4277 & 1 & $0.02 \%(0.00-0.10)$ & 0.00 & $<0.01$ & $N A^{*}$ & & \\
\hline C. andersoni & 6 & 4277 & 100 & $0.25 \%(0.19-0.32)$ & 144.65 & $<0.01$ & 96.5 & & \\
\hline C. suis-like & 1 & 4277 & 2 & $0.05 \%(0.00-0.14)$ & 0.00 & $<0.01$ & $N A^{*}$ & & \\
\hline C. canis & 1 & 4277 & 3 & $0.07 \%(0.01-0.18)$ & 0.00 & $<0.01$ & $\mathrm{NA}^{*}$ & & \\
\hline C. struthionis & 1 & 4277 & 5 & $0.12 \%(0.03-0.25)$ & 0.00 & $<0.01$ & $N A^{*}$ & & \\
\hline C. ubiquitum & 2 & 4277 & 2 & $0.02 \%(0.00-0.10)$ & 0.00 & $<0.01$ & 0.0 & & \\
\hline C. xiaoi & 1 & 4277 & 1 & $0.02 \%(0.00-0.10)$ & 0.00 & $<0.01$ & $\mathrm{NA}^{*}$ & & \\
\hline C. new genotype & 1 & 4277 & 2 & $0.05 \%(0.00-0.14)$ & 0.00 & $<0.01$ & $N A^{*}$ & & \\
\hline
\end{tabular}

$\mathrm{Cl}^{*}$, Confidence interval; $N A^{*}$, not applicable; $P$-value ${ }^{*} P<0.05$ is statistically significant.

TABLE 5 | Sub-group analysis of the prevalence of Cryptosporidium according to geographic location and climate variables.

\begin{tabular}{|c|c|c|c|c|c|c|c|c|c|c|}
\hline Variable & Category & $\begin{array}{l}\text { No. } \\
\text { studies }\end{array}$ & $\begin{array}{c}\text { No. } \\
\text { examined }\end{array}$ & $\begin{array}{c}\text { No. } \\
\text { positive }\end{array}$ & $\%\left(95 \% \mathrm{Cl}^{*}\right)$ & \multicolumn{3}{|c|}{ Heterogeneity } & \multicolumn{2}{|c|}{ Univariate meta-regression } \\
\hline & $30-35^{\circ}$ & 9 & 2858 & 354 & $\begin{array}{c}11.78 \%(5.62- \\
19.77)\end{array}$ & 265.95 & $<0.01$ & 97.0 & & \\
\hline \multirow[t]{3}{*}{ Longitude } & $<95^{\circ}$ & 3 & 1571 & 115 & $6.03 \%(0.37-16.76)$ & 66.98 & $<0.01$ & 97.0 & 0.356 & $-0.126(-0.393$ to \\
\hline & $95-100^{\circ}$ & 7 & 1094 & 198 & $\begin{array}{c}14.22 \%(4.24- \\
28.38)\end{array}$ & 185.29 & $<0.01$ & 96.8 & & $-0.142)$ \\
\hline & $>100^{\circ}$ & 13 & 4536 & 795 & $\begin{array}{c}13.44 \%(5.91- \\
23.35)\end{array}$ & 865.07 & $<0.01$ & 98.6 & & \\
\hline $\begin{array}{l}\text { Precipitation } \\
(\mathrm{mm})\end{array}$ & $<300$ & 5 & 445 & 56 & $\begin{array}{c}11.48 \%(6.00- \\
18.27)\end{array}$ & 12.96 & $<0.01$ & 69.1 & 0.934 & $\begin{array}{c}-0.007(-0.182 \text { to } \\
0.167)\end{array}$ \\
\hline \multirow{2}{*}{ Temperature $\left({ }^{\circ} \mathrm{C}\right)$} & $1-5$ & 11 & 2565 & 414 & $\begin{array}{c}11.09 \%(4.17- \\
20.58)\end{array}$ & 418.38 & $<0.01$ & 97.6 & & \\
\hline & $>5$ & 10 & 2659 & 399 & $\begin{array}{c}11.71 \%(3.60- \\
23.50)\end{array}$ & 538.62 & $<0.01$ & 98.5 & & \\
\hline \multirow[t]{2}{*}{ Humidity } & $<55 \%$ & 12 & 2868 & 365 & $\begin{array}{c}11.09 \%(6.31- \\
16.94)\end{array}$ & 194.38 & $<0.01$ & 94.3 & 0.825 & $\begin{array}{c}-0.019(-0.187 \text { to } \\
0.149)\end{array}$ \\
\hline & $\geq 55 \%$ & 13 & 3539 & 565 & $\begin{array}{c}12.36 \%(4.74- \\
22.80)\end{array}$ & 191.57 & $<0.01$ & 95.3 & & \\
\hline \multirow[t]{2}{*}{ Altitude $(0.1 \mathrm{~m})$} & $<30000$ & 12 & 3646 & 677 & $\begin{array}{c}13.15 \%(5.38- \\
23.60)\end{array}$ & 806.97 & $<0.01$ & 98.5 & 0.850 & $\begin{array}{c}-0.019(-0.219 \text { to } \\
0.181)\end{array}$ \\
\hline & $>30000$ & 16 & 3555 & 433 & $\begin{array}{c}10.40 \%(5.32- \\
16.83)\end{array}$ & 425.07 & $<0.01$ & 96.5 & & \\
\hline
\end{tabular}

$\mathrm{Cl}^{*}$, Confidence interval; $N A^{*}$, not applicable; $P$-value ${ }^{*} P<0.05$ is statistically significant. 


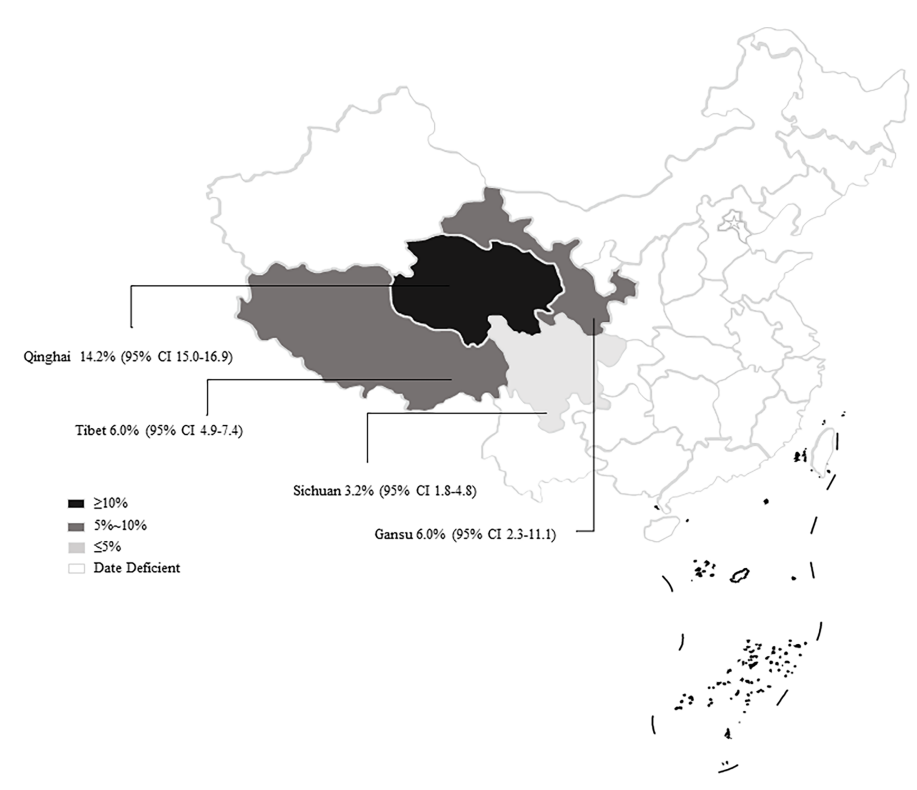

FIGURE 7 | Map of Cryptosporidium prevalence in yaks in China.

spp. infection and prevalence. The forage becomes enriched after the end of cold weather. The body weight and resistance of yaks will increase in the warm weather (Zhou et al., 2020). This may be the reason for the lowest prevalence of Cryptosporidium spp. observed in the seasons with a lower temperature. Thus, we suggest an increased feed should be provided in time to enhance the resistance of yaks in the cold weather.

In this study, the prevalence of Cryptosporidium spp. with Enzyme-linked immunosorbent assay (ELISA) was higher than that with the other three methods in previous reports. ELISA has high specificity and large sample size (Liu et al., 2015; Gong et al., 2020). However, ELISA cannot be used for species typing. In addition, ELISA was rarely used to detect species of parasites (Seema et al., 2014). In this subgroup, there were fewer articles using ELISA to detect Cryptosporidium spp., and the lack of data in this subgroup might lead to a higher prevalence than the other groups, thus resulting in unstable results. The advantages of microscope inspection include simple operation, reasonable price, and easy to capture (Taghipour et al., 2020). Microscopic examination can be used to detect intestinal parasitic infection and shows the presence of pathogens and non-pathogenic parasites, but the specific detection of different Cryptosporidium spp. species is not reliable (Incani et al., 2017; Taghipour et al., 2020). Microscopy also has a low sensitivity which may lead to false positive (Wang et al., 2020). This may be one reason for the high prevalence. IFA has high sensitivity, specificity, and stability for detection of oocysts. The sensitivity is high for even a low oocyst concentration (Ahmed and Panagiotis, 2018). A cross-reaction with fecal yeast during a longer treatment process is one of disadvantages for IFA (Johnston et al., 2003). PCR allows a simultaneous detection of different parasites in a single reaction, which has a higher sensitivity and easier interpretation (Incani et al., 2017). PCR can be used to detect complete DNA and fragments of parasites, and has become the best method for detecting Cryptosporidium spp. (Efrat et al., 2019). Thus, we suggest that the researchers to use the PCR method for detecting Cryptosporidium spp. during epidemiological investigations.

In our meta-analysis $(\mathrm{n}=20)$, there are 5 medium-quality articles and 1 low-quality article. The reason for appearance of medium- or low-quality articles was that most studies had a sample size less than 200 and less than 3 risk factors. It is recommended that researchers should take a large sample size, explore more risk factors, clarify the cause of Cryptosporidium spp. infection, and provide scientific data and theoretical support for the prevention and control of Cryptosporidium spp. infection in yaks.

There were several limitations for our meta-analysis. First, the studies from five databases were limited for obtaining all relevant research data. Second, most of the data were derived from Qinghai province, leading to an uneven data distribution in the northwestern China, and thus affecting the true positive rate. Third, since most of the data show that the yaks are free-range, there is no way to analyze the impact of the feeding mode on Cryptosporidium spp. Finally, the available data for this analysis are limited.

\section{CONCLUSIONS}

The results of this systematic review and meta-analysis using 20 articles showed that Cryptosporidium spp. is common in yaks in China. Different seasons and sampling years had a statistically significant effect on the Cryptosporidium spp. infection in yaks. Yaks under 12 months had a higher prevalence of Cryptosporidium spp. Thus, the protective measures should be strengthened at this age stage. This study provided basic data for the prevention and 
control of cryptosporidiosis in yaks. This may help monitor the prevalence of Cryptosporidium spp. in yaks, prevent and control Cryptosporidium spp. infection in yaks, in order to reduce the risk of Cryptosporidium spp. infection in humans.

\section{DATA AVAILABILITY STATEMENT}

The original contributions presented in the study are included in the article/Supplementary Material. Further inquiries can be directed to the corresponding authors.

\section{ETHICS STATEMENT}

The data regarding the Yaks were collected from five online databases (Chinese National Knowledge Infrastructure (CNKI), VIP Chinese journal database, WanFang Data, PubMed, and ScienceDirect). Written informed consent was obtained from the owners for the participation of their animals in this study.

\section{AUTHOR CONTRIBUTIONS}

$\mathrm{H}-\mathrm{TS}, \mathrm{JJ}$, and $\mathrm{H}-\mathrm{BN}$ were responsible for the idea and concept of the paper. X-YW and WW built the database. H-LG and WW

\section{REFERENCES}

Ahmed, S. A., and Panagiotis, K. (2018). Comparison of Current Methods Used to Detect Cryptosporidium Oocysts in Stools. Int. J. Hyg Environ. Health 221, 743-763. doi: 10.1016/j.ijheh.2018.04.006

Bhat, A. M., Malik, H. U., Wani, N. M., Paul, S., Gupta, S., Dolma, T., et al. (2019). First Report of Cryptosporidium Sp. Infection in Sheep Population of Ladakh, India. J. Parasit Dis. 43 (3), 513-516. doi: 10.1007/s12639-019-01119-1

Deng, L., Chai, Y. J., Luo, R., Yang, L. L., Yao, J. X., Zhong, Z. J., et al. (2020). Occurrence and Genetic Characteristics of Cryptosporidium Spp. And Enterocytozoon Bieneusi in Pet Red Squirrels (Sciurus Vulgaris) in China. Sci. Rep. 10 (1), 1026. doi: 10.1038/s41598-020-57896-w

Desai, A. N. (2020). Cryptosporidiosis. JAMA. 323, 288. doi: 10.1001/ jama.2019.18691

Efrat, G. S., Said, A., Tamar, G., Esther, M., Avi, O., Maya, A., et al. (2019). The Prevalence of Cryptosporidium Among Children Hospitalized Because of Gastrointestinal Symptoms and the Efficiency of Diagnostic Methods for Cryptosporidium. Am. J. Trop. Med. Hyg. 101, 160-163. doi: 10.4269/ajtmh.19-0057

Fan, S. R., Fan, G. Z., Dong, Y. P., and Zhou, D. W. (2011). Discussion on the Division Method of Four Seasons in Qinghai Tibet Plateau. Plateau Mountain Meteorol Res. 31, 1-11.

Fu, Y. X., Zhao, Z. F., and Chen, B. L. (2018). Research on Spatial Distribution of Grassland Resources in China. J. Sci. Technol. Inf. 16, 68-69.

Gao, M. (2012). Studies on the Species of Bovine Coccidiosis and Genotyping of Cryptosporidium Andersoni in Guanzhong Area. Northwest A\&F Univ. 1, 1-60.

General Office of the State Council (2012). National Medium-and Long-Term Plan for Prevention and Control of Animal Disease-2020). Heilongjiang J. Anim. Vet. Sci. 33 (7), 26-30.

Gong, C., Cao, X. F., Deng, L., Li, W., Huang, X. M., Lan, J. C., et al. (2017). Epidemiology of Cryptosporidium Infection in Cattle in China: A Review. Parasite 24:1. doi: 10.1051/parasite/2017001

Gong, Q. L., Li, J., Li, D., Tian, T., Leng, X., Li, J. M., et al. (2020). Seroprevalence of Toxoplasma Gondii in Cattle in China From 2010 to 2019: A Systematic Review analyzed the data. H-LG wrote the manuscript. J-HL, JJ, and $\mathrm{X}-\mathrm{YW}$ critically reviewed and revised the manuscript. All authors contributed to the article and approved the submitted version.

\section{FUNDING}

This work was supported by the Wild Animal Disease Monitoring and Early Warning System Maintenance Project (2130211), and the Research Foundation for Distinguished Scholars of Qingdao Agricultural University (665-1120046).

\section{ACKNOWLEDGMENTS}

We thank Dr. Chuang Lyu (Shandong New Hope Liuhe Group Co., Ltd. \& Qingdao Jiazhi Biotechnology Co., Ltd., Qingdao, China) for critically revising the manuscript.

\section{SUPPLEMENTARY MATERIAL}

The Supplementary Material for this article can be found online at: https://www.frontiersin.org/articles/10.3389/fcimb.2021. 770612/full\#supplementary-material and Meta-Analysis. Acta Trop. 211, 105439. doi: 10.1016/j.actatropica. 2020.105439

Guyatt, G. H., Oxman, A. D., Vist, G. E., Kunz, R., Falck-Ytter, Y., Alonso-Coello, P., et al. (2008). GRADE: An Emerging Consensus on Rating Quality of Evidence and Strength of Recommendations. BMJ. 336, 924-926. doi: 10.1136/ bmj.39489.470347.AD

Guy, R. A., Yanta, C. A., Muchaal, P. K., Rankin, M. A., Thivierge, K., Lau, R., et al. (2021). Molecular Characterization of Cryptosporidium Isolates From Humans in Ontario, Canada. Parasit Vectors. 14 (1), 69. doi: 10.1186/s13071-020-04546-9

Huang, J., Yue, D., Qi, M., Wang, R., Zhao, J., Li, J., et al. (2014). Prevalence and Molecular Characterization of Cryptosporidium Spp. And Giardia Duodenalis in Dairy Cattle in Ningxia, Northwestern China. BMC Vet. Res. 10:292. doi: 10.1186/s12917-014-0292-6

Ikiroma, I. A., and Polloc, K. G. (2021). Influence of Weather and Climate on Cryptosporidiosis-A Review. Zoonoses Public Health 68, 285-298. doi: $10.1111 /$ zph.12785

Incani, R. N., Ferrer, E., Hoek, D., Ramak, R., Roelfsema, J., Mughini-Gras, L., et al. (2017). Diagnosis of Intestinal Parasites in a Rural Community of Venezuela: Advantages and Disadvantages of Using Microscopy or RT-PCR. Acta Trop. 167, 64-70. doi: 10.1016/j.actatropica.2016.12.014

Jagai, J. S., Castronovo, D. A., Monchak, J., and Naumova, E. N. (2009). Seasonality of Cryptosporidiosis: A Meta-Analysis Approach. Environ. Res. 109 (4), 465-478. doi: 10.1016/j.envres.2009.02.008

Johnston, S. P., Ballard, M. M., Beach, M. J., Causer, L., and Wilkins, P. P. (2003). Evaluation of Three Commercial Assays for Detection of Giardia and Cryptosporidium Organisms in Fecal Specimens. J. Clin. Microbiol. 41, 623 626. doi: 10.1128/JCM.41.2.623-626.2003

Karanis, P., Plutzer, J., Halim, N. A., Igor, K., Nagasawa, H., Ongerth, J., et al. (2007). Molecular Characterization of Cryptosporidium From Animal Sources in Qinghai Province of China. Parasitol Res. 101, 1575-1580. doi: 10.1007/ s00436-007-0681-x

Lan, D., Ji, W., Xiong, X., Liang, Q., Yao, W., Mipam, T. D., et al. (2020). Population Genome of the Newly Discovered Jinchuan Yak to Understand its 
Adaptive Evolution in Extreme Environments and Generation Mechanism of the Multirib Trait. Integr. Zool. 16 (5), 685-695. doi: 10.1111/1749-4877.12484

Li, Z. (2017). Safety Analysis and Potential Hazard Control of Yak Feeding Inputs. Southwest Minzu Univ. 2017, 1-110.

Li, P., Cai, J., Cai, M., Wu, W., Li, C., Lei, M., et al. (2016a). Distribution of Cryptosporidium Species in Tibetan Sheep and Yaks in Qinghai, China. Vet. Parasitol. 215, 58-62. doi: 10.1016/j.vetpar.2015.11.009

Li, K., Li, Z. X., Zeng, Z. B., Li, A. Y., Mehmood, K., Shahzad, M., et al. (2020b). Prevalence and Molecular Characterization of Cryptosporidium Spp. In Yaks (Bos Grunniens) in Naqu, China. Microb. Pathog. 144, 104190. doi: 10.1016/ j.micpath.2020.104190

Li, K., Nader, S. M., Zhang, X., Ray, B. C., Kim, C. Y., Das, A., et al. (2019). Novel Lactate Dehydrogenase Inhibitors With In Vivo Efficacy Against Cryptosporidium Parvum. PloS Pathog. 15, e1007953. doi: 10.1371/ journal.ppat.1007953

Li, X., Ni, H. B., Ren, W. X., Jiang, J., Gong, Q. L., and Zhang, X. X. (2020a). Seroprevalence of Toxoplasma Gondii in Horses: A Global Systematic Review and Meta-Analysis. Acta Trop. 201, 105222. doi: 10.1016/j.actatropica.2019

Liu, Q., Wang, Z. D., Huang, S. Y., and Zhu, X. Q. (2015). Diagnosis of Toxoplasmosis and Typing of Toxoplasma Gondii. Parasit Vectors. 8, 292. doi: 10.1186/s13071-015-0902-6

Li, F. H., Wang, H. Y., Zhang, Z. J., Li, J. Q., Wang, C. R., Zhao, J. F., et al. (2016b). Prevalence and Molecular Characterization of Cryptosporidium Spp. And Giardia Duodenalis in Dairy Cattle in Beijing, China. Vet. Parasitol. 219, 6165. doi: 10.1016/j.vetpar.2016.01.023

Li, N., Xiao, L., Alderisio, K., Elwin, K., Cebelinski, E., Chalmers, R., et al. (2014). Subtyping Cryptosporidium Ubiquitum, a Zoonotic Pathogen Emerging in Humans. Emerg. Infect. Dis. 20, 217-224. doi: 10.3201/eid2002.121797

Lou, Z. L. (2016). Epidemiological Investigation and Genotyping of Toxoplasma Gondii, Microsporidium and Cryptosporidium in Cultured Arctic Foxes in Eastern China (Jilin Agricultural University), 1-67 (in Chinese).

Ma, L. Q., Cai, Q. G., and Lu, Y. (2010). Serological Investigation on Cryptosporidiosis of Lambs in Qinghai Province. Chin. J. Vet. Med. 2010, 55-56.

Ma, J. B., Cai, J. Z., Ma, J. W., Feng, Y. Y., and Xiao, L. H. (2014b). Occurrence and Molecular Characterization of Cryptosporidium Spp. In Yaks (Bos Grunniens) in China. Vet. Parasitol. 202 (3-4), 113-118. doi: 10.1016/j.vetpar.2014.03.030

Ma, L. Q., Sotiriadou, I., Cai, Q. G., Karanis, G., Wang, G. P., Wang, G. H., et al. (2014a). Detection of Cryptosporidium and Giardia in Agricultural and Water Environments in the Qinghai Area of China by IFA and PCR. Parasitol Res. 113, 3177-3184. doi: 10.1007/s00436-014-3979-5

Ma, L. Q., Wang, G. P., Lu, Y., Cai, Q. G., Wang, G. H., Li, X. P., et al. (2013). Molecular Characteristics of Cryptosporidium in Qinghai Province. Chin. Qinghai J. Anim. Vet. Sci. 2013, 1-3.

Ma, L. Q., Zhang, X. Y., Jian, Y. N., Li, X. P., Wang, G. P., Hu, Y., et al. (2019). Detection of Cryptosporidium and Giardia in the Slaughterhouse, Sewage and River Waters of the Qinghai Tibetan Plateau Area (QTPA), China. Parasitol Res. 118 (7), 2041-2051. doi: 10.1007/s00436-019-06330-w

Mi, R., Wang, X., Li, C., Huang, Y., Zhou, P., Li, Z., et al. (2013). Prevalence and Genetic Characterization of Cryptosporidium in Yaks in Qinghai Province of China. PloS One 8, e74985. doi: 10.1007/s00436-018-5861-3

Murakoshi, F., Xiao, L., Matsubara, R., Sato, R., Kato, Y., Sasaki, T., et al. (2012). Molecular Characterization of Cryptosporidium Spp. In Grazing Beef Cattle in Japan. Vet. Parasitol. 187 (1-2), 123-128. doi: 10.1016/j.vetpar.2011.12.011

Ni, H. B., Gong, Q. L., Zhao, Q., Li, X. Y., and Zhang, X. X. (2020). Prevalence of Haemophilus Parasuis "Glaesserella Parasuis" in Pigs in China: A Systematic Review and Meta-Analysis. Prev. Vet. Med. 182, 105083. doi: 10.1016/ j.prevetmed.2020.105083

Niu, X. Y., and Ma, L. Q. (2007). Serological Investigation of Sheep Cryptosporidiosis in Haixi Area of Qinghai Province. Chin. J. Vet. Med. 2007, 55-56.

Ouakli, N., Belkhiri, A., de Lucio, A., Köster, P. C., Djoudi, M., Dadda, A., et al. (2018). Cryptosporidium-Associated Diarrhoea in Neonatal Calves in Algeria. Vet. Parasitol Reg. Stud. Rep. 12, 78-84. doi: 10.1016/j.vprsr.2018.02.005

Pumipuntu, N., and Piratae, S. (2018). Cryptosporidiosis: A Zoonotic Disease Concern. Vet. World. 11 (5), 681-686. doi: 10.14202/vetworld.2018.681-686

Qi, M., Cai, J. Z., Wang, R. J., Li, J. Q., Jian, F. C., Huang, J. Y., et al. (2015). Molecular Characterization of Cryptosporidium Spp. And Giardia Duodenalis
From Yaks in the Central Western Region of China. BMC Microbiol. 15, 108 doi: 10.1186/s12866-015-0446-0

Qin, S. Y., Zhang, X. X., Zhao, G. H., Zhou, D. H., Yin, M. Y., Zhao, Q., et al. (2014). First Report of Cryptosporidium Spp. In White Yaks in China. Parasit Vectors. 7, 230. doi: 10.1186/1756-3305-7-230

Ryan, U., Zahedi, A., and Paparini, A. (2016). Cryptosporidium in Humans and Animals-A One Health Approach to Prophylaxis. Parasite Immunol. 38, 535547. doi: 10.1111/pim.12350

Sareyyüpoğlu, B., Gülyaz, V., Çokçalışkan, C., Ünal, Y., Çökülgen, T., Uzunlu, E., et al. (2019). Effect of FMD Vaccination Schedule of Dams on the Level and Duration of Maternally Derived Antibodies. Vet. Immunol. Immunopathol. 217, 109881. doi: 10.1016/j.vetimm.2019.109881

Seema, M., Madhu, S., Uma, C., and Aparna, Y. (2014). Comparison of ELISA and Microscopy for Detection of Cryptosporidium in Stool. J. Clin. Diagn. Res. 8, DC07-DC08. doi: 10.7860/JCDR/2014/9713.5088

Taghipour, A., Olfatifar, M., Bahadory, S., Godfrey, S. S., Abdoli, A., Khatami, A., et al. (2020). The Global Prevalence of Cryptosporidium Infection in Dogs: A Systematic Review and Meta-Analysis. Vet. Parasitol. 281, 109093. doi: 10.1016/j.vetpar.2020.109093

Wang, W., Gong, Q. L., Zeng, A., Li, M. H., Zhao, Q., and Ni, H. B. (2020). Prevalence of Cryptosporidium in Pigs in China: A Systematic Review and Meta-Analysis. Transbound Emerg. Dis. 68, 1400-1413. doi: 10.1111/ tbed.13806

Wang, Y., Shen, Y., Liu, H., Yin, J., Zhang, X. T., Gong, A. Y., et al. (2019a). Induction of Inflammatory Responses in Splenocytes by Exosomes Released From Intestinal Epithelial Cells Following Cryptosporidium Parvum Infection. Infect. Immun. 87 (4), e00705-e00718. doi: 10.1128/IAI.00705-18

Wang, G. P., Wang, G. H., Li, X. P., Zhang, X. Y., Karanis, G., Jian, Y. N., et al. (2018). Prevalence and Molecular Characterization of Cryptosporidium Spp. And Giardia Duodenalis in 1-2-Month-Old Highland Yaks in Qinghai Province, China. Parasitol Res. 117, 1793-1800. doi: 10.1007/s00436-0185861-3

Wang, R., Wang, H., Sun, Y., Zhang, L., Jian, F., Qi, M., et al. (2011). Characteristics of Cryptosporidium Transmission in Preweaned Dairy Cattle in Henan, China. J. Clin. Microbiol. 9 (3), 1077-1082. doi: 10.1128/ JCM.02194-10

Wang, R., Zhao, G., Gong, Y., and Zhang, L. (2017). Advances and Perspectives on the Epidemiology of Bovine Cryptosporidium in China in the Past 30 Years. Front. Microbiol. 8:1823. doi: 10.3389/fmicb.2017.01823

Wang, K., Zhu, C. Z., Zhao, G. M., Hao, W. M., Tian, W., and Shen, M. Y. (2019b). Breed and Distribution of Yak in China. Chin. J. Anim. Sci., 168-171.

Wei, X. Y., Gong, Q. L., Zeng, A., Wang, W., Wang, Q., and Zhang, X. X. (2021). Seroprevalence and Risk Factors of Toxoplasma Gondii Infection in Goats in China From 2010 to 2020: A Systematic Review and Meta-Analysis. Prev. Vet. Med. 186, 105230. doi: 10.1016/j.prevetmed.2020

Wei, D., Xu, R., Tenzin, T., Wang, Y., and Wang, Y. (2015). Considerable Methane Uptake by Alpine Grasslands Despite the Cold Climate: In Situ Measurements on the Central Tibetan Platea-2013. Glob Chang Biol. 21, 777-788. doi: $10.1111 / \mathrm{gcb} .12690$

Wen, Z. Z. (1988). Investigation on Age Distribution of Yak Population and Analysis of Intraspecies Structure. J. Domest. Anim. Ecol. 1988 (02), $16-22+38$.

Widmer, G. (2009). Meta-Analysis of a Polymorphic Surface Glycoprotein of the Parasitic Protozoa Cryptosporidium Parvum and Cryptosporidium Hominis. Epidemiol. Infect. 137, 1800-1808. doi: 10.1017/S0950268809990215

Xiao, L., Fayer, R., Ryan, U., and Upton, S. J. (2004). Cryptosporidium Taxonomy: Recent Advances and Implications for Public Health. Clin. Microbiol. Rev. 17 (1), 72-97. doi: 10.1128/CMR.17.1.72-97.2004

Yildirim, A., Adanir, R., Inci, A., Yukari, B. A., Duzlu, O., Onder, Z., et al. (2020). Prevalence and Genotyping of Bovine Cryptosporidium Species in the Mediterranean and Central Anatolia Region of Turkey. Comp. Immunol. Microbiol. Infect. Dis. 69, 101425. doi: 10.1016/j.cimid.2020.101425

Zhang, X. H. (2010). SWOT Analysis of Ecotourism Development in Qinghai Province. J. Landscape Res. 2, 42-44+48.

Zhang, W., Wang, R., Yang, F., Zhang, L., Cao, J., Zhang, X., et al. (2013). Distribution and Genetic Characterizations of Cryptosporidium Spp. In PreWeaned Dairy Calves in Northeastern China's Heilongjiang Province. PloS One 8, e54857. doi: 10.1371/journal.pone.0054857 
Zhang, Q. X., Zhang, Z. C., Ai, S. T., Wang, X. Q., Zhang, R. Y., and Duan, Z. Y. (2019). Cryptosporidium Spp., Enterocytozoon Bieneusi, and Giardia Duodenalis From Animal Sources in the Qinghai-Tibetan Plateau Area (QTPA) in China. Comp. Immunol. Microbiol. Infect. Dis. 67, 101346. doi: 10.1016/j.cimid.2019.101346

Zhou, J., Yue, S., Peng, Q., Wang, L., Wang, Z., and Xue, B. (2020). Metabonomic Responses of Grazing Yak to Different Concentrate Supplementations in Cold Season. Anim. (Basel) 10, 1595. doi: 10.3390/ani10091595

Conflict of Interest: The authors declare that the research was conducted in the absence of any commercial or financial relationships that could be construed as a potential conflict of interest.
Publisher's Note: All claims expressed in this article are solely those of the authors and do not necessarily represent those of their affiliated organizations, or those of the publisher, the editors and the reviewers. Any product that may be evaluated in this article, or claim that may be made by its manufacturer, is not guaranteed or endorsed by the publisher.

Copyright $\odot 2021$ Geng, Ni, Li, Jiang, Wang, Wei, Zhang and Sun. This is an openaccess article distributed under the terms of the Creative Commons Attribution License (CC BY). The use, distribution or reproduction in other forums is permitted, provided the original author(s) and the copyright owner(s) are credited and that the original publication in this journal is cited, in accordance with accepted academic practice. No use, distribution or reproduction is permitted which does not comply with these terms. 